\title{
Efficacy of a ketogenic diet with concomitant intranasal perillyl alcohol as a novel strategy for the therapy of recurrent glioblastoma
}

\author{
JULIANA GUIMARÃES SANTOS ${ }^{1}$, WANISE MARIA SOUZA DA CRUZ ${ }^{2}$, AXEL H. SCHÖNTHAL ${ }^{3}$, \\ MARCELA D'ALINCOURT SALAZAR ${ }^{4}$, CRISTINA ASVOLINSQUE PANTALEÃO FONTES ${ }^{5}$, \\ THEREZA QUIRICO-SANTOS $^{6}$ and CLOVIS ORLANDO DA FONSECA ${ }^{7}$
}

\footnotetext{
${ }^{1}$ Graduate Program in Medical Sciences, Fluminense Federal University, Niteroi, Rio de Janeiro 24033-900;

${ }^{2}$ Department of Nutrition, Fluminense Federal University, Niteroi, Rio de Janeiro 24020-150, Brazil;

${ }^{3}$ Department of Molecular Microbiology and Immunology, Keck School of Medicine, University of Southern California, Los Angeles, CA 90089; ${ }^{4}$ Department of Experimental Therapeutics, Beckman Research

Institute of City of Hope, Duarte, CA 91010, USA; ${ }^{5}$ Service of Radiology, Department of Radiology,

Antonio Pedro University Hospital, Fluminense Federal University, Niteroi, Rio de Janeiro 24033-900;

${ }^{6}$ Department of Cellular and Molecular Biology, Institute of Biology, Fluminense Federal University, Niteroi,

Rio de Janeiro 24210-130; ${ }^{7}$ Service of Neurosurgery, Department of General and Specialized Surgery, Antonio Pedro University Hospital, Fluminense Federal University, Niteroi, Rio de Janeiro 24033-900, Brazil
}

Received March 26, 2017; Accepted June 9, 2017

DOI: $10.3892 / \mathrm{ol} .2017 .7362$

\begin{abstract}
It has been hypothesized that persistent ketotic hypoglycemia represents a potential therapeutic strategy against high-grade gliomas. Perillyl alcohol $(\mathrm{POH})$ is a non-toxic, naturally-occurring, hydroxylated monoterpene that exhibits cytotoxicity against temozolomide-resistant glioma cells, regardless of O6-methylguanine-methyltransferase promoter methylation status. The present study aimed to evaluate the toxicity and therapeutic efficacy of intranasal $\mathrm{POH}$ when administered in combination with a ketogenic diet (KD) program for the treatment of patients with recurrent glioblastoma. The 32 enrolled patients were divided into two groups, KD or standard diet, with intranasal $\mathrm{POH}$ treatment $(\mathrm{n}=17$ and $\mathrm{n}=15$, respectively). The nutritional status and anthropometric parameters of the patients were measured. Patients that adhered
\end{abstract}

Correspondence to: Dr Juliana Guimarães Santos, Graduate Program in Medical Sciences, Fluminense Federal University, 303 Avenue Marquês do Paraná, Niteroi, Rio de Janeiro 24033-900, Brazil

E-mail: julianaguima86@gmail.com

Professor Clovis Orlando da Fonseca, Service of Neurosurgery, Department of General and Specialized Surgery, Antonio Pedro University Hospital, Fluminense Federal University, 303 Avenue Marquês do Paraná, Niteroi, Rio de Janeiro 24033-900, Brazil E-mail: clovis.orlando@uol.com.br

Key words: ketogenic diet, perillyl alcohol, intranasal administration, recurrent glioblastoma, combination therapy to the KD maintained a strict dietary regimen, in addition to receiving $55 \mathrm{mg} \mathrm{POH}$ four times daily, in an uninterrupted administration schedule for three months. Neurological examination and magnetic resonance imaging analysis were used to monitor disease progression. A total of 9/17 patients in the KD group survived and maintained compliance with the KD. After three months of well-tolerated treatment, a partial response (PR) was observed for $77.8 \%(7 / 9)$ of the patients, stable disease (SD) in $11.1 \%(1 / 9)$ and $11.1 \%(1 / 9)$ presented with progressive disease (PD). Among the patients assigned to the standard diet group, the PR rate was $25 \%$ (2/8 patients), SD $25 \%(2 / 8)$ and PD 50\% (4/8 patients). The patients assigned to the KD group presented with reduced serum lipid levels and decreased low-density lipoprotein cholesterol levels. These results are encouraging and suggest that KD associated with intranasal $\mathrm{POH}$ may represent a viable option as an adjunct therapy for recurrent GBM.

\section{Introduction}

Glioblastoma (GBM) is a highly aggressive primary brain cancer that is difficult to treat. The current standard of care consists of surgery, followed by radiation and chemotherapy with the alkylating agent temozolomide (TMZ) (1). However, the therapeutic benefit of TMZ is restricted to the patients presenting with epigenetic silencing of the O6-methylguanine-DNA methyltransferase (MGMT) DNA repair gene in the tumor (2). Otherwise, high expression levels of MGMT allow tumor cells to repair O6-guanine subsequent to methylation by TMZ, thereby preventing drug-induced DNA double-strand breaks and tumor cell death. As a consequence, median overall survival time differs depending on MGMT promoter methylation status, 
with 15 months for patients with an unmethylated promoter and 22 months for those with the methylated promoter status (3). In either case, however, (89-97\%) of patients succumb to the disease within 5 years, as even for patients initially responding favorably to TMZ, the effectiveness wanes over time as tumors develop drug resistance (4).

A newly-approved vaccine-based therapy, rindopepimut (CDX-110), has demonstrated further extension of median survival time; however, this regimen is only applicable to the $30 \%$ of GBM patients who are positive for the epidermal growth factor receptor variant EGFRvIII (5). An approved treatment for the recurrent setting is the angiogenesis inhibitor bevacizumab (6). Although it improves the outcome for the majority of GBM patients, the duration of benefit is limited and the survival rate from the time of initial bevacizumab progression is poor $(7,8)$. The latest therapy to be approved is the alternating electric fields generator-based NovoTTF therapy, (9) which has been demonstrated to extend median overall survival time by $\sim 5$ months (10). Regardless of the treatment regimen, however, patients with GBM continue to exhibit a dismal prognosis and novel methods of treatment are urgently required.

In one novel approach, the intranasal delivery of perillyl alcohol (POH), a naturally occurring monoterpene related to limonene (11), was investigated in two clinical studies with grade III and grade IV glioma patients (12-14). POH was administered via a lightweight nebulizer and nose mask up to four times daily on a continuous basis. The results were highly promising, indicating therapeutic activity along with good tolerance and no long-term central nervous system or systemic severe adverse events, even following years of daily administration (12-14). POH activity was also noted in recurrent patients, i.e., patients who had become unresponsive to standard of care, and $19 \%$ of all patients remained in clinical remission following 4 years of continuous, exclusive treatment with intranasal POH $(13,14)$. As of April 2017, a clinical trial is currently underway in the United States that aims to investigate the safety and efficacy of intranasal NEO100, a highly purified version of perillyl alcohol, in patients with recurrent grade IV glioma (NCT02704858).

Another newly emerging principle being investigated for the treatment of GBM is the ketogenic diet (KD). Besides being a lifestyle choice or a method for weight loss, in medicine the KD is prescribed primarily as a treatment for refractory epilepsy in children (15). Based on its high-fat/low-carbohydrate content, the diet forces the body to metabolize fats and ketone bodies rather than glucose; the state of ketosis is hypothesized to reduce the frequency of epileptic seizures (16).

In the context of brain tumor treatment, the KD is expected to provide two main benefits. First, as epilepsy commonly develops among GBM patients (17), it may aid a reduction in the severity and number of epileptic episodes and alleviate the requirement for anti-epileptic drugs, which are associated with detrimental neuropsychological effects and often interfere with the therapeutic regimen $(18,19)$. Second, KD may increase the efficacy of cancer therapy. Tumor development is frequently associated with a metabolic alteration, in which cells switch from aerobic respiration towards increased glycolysis and lactate production, despite the availability of oxygen (20). This so-called Warburg effect (aerobic glycolysis) leads to the increased consumption of glucose and the inability of the affected cells to utilize ketone bodies as an energy source (21). In response to $\mathrm{KD}$, it is hypothesized that tumor cells will starve, or revert to normal mitochondrial activity and respiration, altogether resulting in decreased proliferation and metastatic ability, along with a heightened sensitivity to chemotherapy (22-24).

Previous preclinical studies demonstrated notable benefits of the KD in a variety of animal tumor models, including in malignant brain tumors (25) and in the meta-analysis by Klement et al (26). For example, among groups of mice with luciferase-labeled GL261 glioma cells implanted into their brains, median survival time was significantly extended in mice on a KD compared with mice on a standard diet; in addition, when such mice received two fractions of radiation ( $2 \times 4$ Gy of whole brain radiation), the bioluminescent signals from the intracranial tumors was diminished below the detectable limit in $82 \%$ of the mice, and no signs of tumor recurrence were observed for $>200$ days (27).

Encouraging results from the use of a KD were also reported in patients, and a small number of case studies have indicated a benefit of the KD in patients with malignant glioma, as reviewed by Maroon et al (28). It was consistently reported that patients undergoing the KD presented with reduced blood glucose levels and beneficial therapeutic responses, including the transient disappearance of the brain tumor tissue (29-31). Thus, these case reports, along with numerous anecdotal observations, presented encouraging signs of metabolic reprogramming by the KD, along with the increased efficacy of standard of care treatment for malignant glioma $(23,29)$. This preliminary evidence of the benefit of $\mathrm{KD}$ for cancer therapy is currently being validated in ongoing, controlled clinical trials, which include patients with malignant glioma (25).

At the molecular level, reduced glucose availability may cause endoplasmic reticulum (ER) stress, which triggers the unfolded protein response (UPR) cellular process, consisting of an interplay of antagonistic mechanisms; low to moderate activity is cell protective and supports chemoresistance, but more severe conditions aggravate these mechanisms to the point where protective efforts are abandoned and the cell death program is induced instead (32). As tumor cells frequently experience chronic stress conditions (due to hypoxia, hypoglycemia, acidification, oxidative stress, etc.), the protective components of their ER stress response are continuously engaged and thus, less able to neutralize additional insults that tax the ER stress response $(33,34)$. Of note, the ER stress/UPR process has been described as a potential therapeutic target in $\operatorname{GBM}(35,36)$ and this cellular mechanism has been demonstrated to be targeted by POH in GBM cells in vitro (37).

It is thus conceivable that the concerted effect of KD-induced hypoglycemia together with $\mathrm{POH}$-induced responses may trigger severely aggravated ER stress, resulting in tumor cell apoptosis $(38,39)$. Therefore, the present study combined a KD with the intranasal delivery of $\mathrm{POH}$, and investigated the effects of this novel combination in a cohort of 32 patients who had previously relapsed from standard of care treatment. 


\section{Patients and methods}

Patient selection and treatment. The present study was approved by the Fluminense Federal University (Niteroi, Brazil; UFF-CAAE: 14613313.8.0000.5243), and was performed at the Antonio Pedro University Hospital, Fluminense Federal University. Each patient signed a written informed consent prior to enrolling in the clinical trial of KD with intranasal delivery of $\mathrm{POH}$. $\mathrm{POH}$ was formulated for delivery by inhalation and the preparation was supplied by the Multidisciplinary Laboratory of Pharmaceutical Sciences at Rio de Janeiro Federal University (Rio de Janeiro, Brazil), according to the Brazilian patent no. PI 0107262-5. KD was administered concomitantly with the daily inhalation of $\mathrm{POH}$ for three months. POH (55 mg; $0.3 \% \mathrm{v} / \mathrm{v})$ was administered by inhalation 4 times per day, totaling $266.8 \mathrm{mg} /$ day.

For patients to be included in the trial, a histopathological diagnosis of malignant glioma was required. This was performed during their initial surgery. Additionally, all patients presented with relapsed GBM, had no further standard therapeutic options, were aged $>18$ years, had measurable contrast-enhancing tumor on magnetic resonance imaging (MRI), Karnofsky performance scale of $\geq 70 \%$ or higher, adequate bone marrow function, white blood cell count of $\geq 3,000 / 1$, absolute neutrophil count of $\geq 1,500 / 1$, platelet count of $\geq 100,000 / 1$, hemoglobin of $\geq 8.0 \mathrm{~g} / \mathrm{dl}$, bilirubin of $\geq 0.3 \mathrm{mg}$.

$K D$ details and monitoring. The anthropometric and biochemical status of each patient was assessed at the time of inclusion in the study. The KD was prescribed according to the following distribution: Energy, $25 \mathrm{kcal} / \mathrm{kg}$; protein, $1.5 \mathrm{~g} / \mathrm{kg} ; 25 \%$ of total calories from carbohydrate, $50 \%$ from lipids; cholesterol, $\leq 200 \mathrm{mg} /$ day; saturated fat, $<7 \%$; polyunsaturated fat, $<10 \%$; monounsaturated fat, $<20 \%$ and fiber, 20-30 g/day. Analysis was performed with urinary ketones as it was cheaper and simple for the patients to perform. Participants received urinalysis strips and instructions for use of the test strip. They were instructed to perform the measurement 3 times a week and report the results to the researchers weekly. Levels of ketone bodies in the urine of $5-15 \mathrm{mg} / \mathrm{dl}$ were considered to confirm adherence to the KD. Adverse effects, including nausea, weakness, tremors, mood swings, headaches, dizziness and drowsiness were monitored through patient/family reporting by phone contact, and reporting on medical records during the visit.

Patient imaging. Imaging examinations were performed at a range of institutions, using a 1.5 Tesla magnetic resonance imaging (MRI) scanner with a brain coil device. All examinations were included in the complete routine for MRI evaluation with multiplanar $\mathrm{T} 1 \mathrm{~W}$ acquisitions prior and subsequent to the venous administration of paramagnetic contrast with volumetric series in $3 \mathrm{D}, \mathrm{T} 2$, T2/fluid-attenuated inversion recovery (FLAIR), diffusion and susceptibility weighted imaging. Magnetic resonance (MR) proton spectroscopy and MR perfusion were also performed. Images of the MR examinations were analyzed by two radiologists with experience in neuroradiology that produced a consensus diagnosis using a workstation with a picture archiving and communication system. The observing radiologists were aware of the GBM diagnosis and follow-up data. The image analysis focused on the following characteristics: Lesion distribution and volume, signal intensity and T2/FLAIR imaging characteristics, perilesional edema, diffusion restriction patterns, and contrast enhancement.

Assessment of outcome. At the beginning of an evaluation, lesions were measured in order to provide bases for comparison. Response assessment and evaluation criteria for target lesions were as follows: Complete response (CR), all target lesions disappeared during the course of treatment; partial response (PR), decreases of $\geq 30 \%$ were noted in the lesion with the largest diameter; stable disease (SD), there was no significant decrease or increase in the size of target lesions; progressive disease (PD), there was an increase of $\geq 20 \%$ in the largest diameter of targeted lesions or the appearance of a new lesion. Other parameters evaluated were: Peritumoral edema, neurological stability, corticosteroid requirement and general improvement of condition, including reduced frequency of seizures, weight loss, reduced serum lipid levels and low density lipoprotein cholesterol (LDL-C), reduced body fat and increase in lean muscle mass.

Statistical methods. To evaluate the tumor area associated with brain edema before and after dietary intervention in the groups, statistical analysis was performed using a unilateral signal test. A paired Student's t-test was performed to compare normally distributed biochemical parameters, whereas a signal test was used for non-normal parameters, within each treatment group. Comparisons between groups at equivalent timepoints were made with a Student's t-test for normally distributed data and a Mann-Whitney test for non-normal parameters. Statistical tests were performed using SPSS software version 18.0 (SPSS, Inc., Chicago, IL, USA). $\mathrm{P}<0.05$ was considered to indicate a statistically significant difference. The data were graphically presented using box plots.

\section{Results}

Patient characteristics. A total of 32 patients with recurrent GBM were enrolled. A total of 17 were allocated to the KD treatment group (range, 31-61; median, 53 years old) and 15 to the control group (standard diet; range, 27-55 years; median, 48 years old). A total of 17 patients completed the study, including 9 patients in the KD group and 8 patients in the control group. Of the 15 patients that did not complete the study, 12 succumbed to their disease and 3 were excluded due to diet non-adherence. Before entering the clinical trial, all patients had received prior conventional therapy (including surgery, chemotherapy and radiotherapy; Table I). No adverse effects, including nausea, weakness, tremors, mood swings, headaches, dizziness or drowsiness, were observed in the study participants.

Clinical outcomes. Following three months of adherence to the treatment in the KD group, PR was observed in $77.8 \%(7 / 9)$ patients, characterized by the reduction of MRI tumor size and peritumoral edema (Fig. 1), neurological stability, reduced corticosteroid requirement, and the general improvement of condition. The corticosteroid was maintained during the trial 
for all patients, but the dose was decreased in the patients who presented with an improvement of clinical status (5/9 patients). PD was observed in $11.1 \%$ (1/9) patients, with an increased tumor size or the appearance of a new lesion in MRI. Among the patients of the control group, PR was noted in $25.0 \%$ (2/8 patients), and $\mathrm{PD}$ was observed in $50.0 \%$ (4/8 patients). The imaging data demonstrated that tumor area was significantly smaller in the KD group at 90 days compared with the baseline ( $\mathrm{P}=0.035)$, and not in the control group ( $\mathrm{P}=0.687$; Fig. 2).

There was a statistically significant difference $(\mathrm{P}=0.003)$ between the total cholesterol level in the initial and final measurements of the KD group, with values significantly lower at 90 days (Fig. 3). There was a statistically significant difference $(\mathrm{P}=0.0002)$ between LDL-C at the initial and final time in treated patients, with higher values at the onset. Test levels of triglycerides also exhibited statistically significant differences $(\mathrm{P}=0.004)$ between the initial and final time in the KD group, with higher values at baseline. The same comparisons of total cholesterol, LDL-C and triglycerides in the standard diet group revealed no statistically significant differences between the baseline and 90 days. In the comparisons between the groups (KD and control) at equivalent timepoints, significant differences were observed with regard to total cholesterol and triglycerides between the groups at the initial time $(\mathrm{P}=0.013$ and $\mathrm{P}=0.036$, respectively); however, there was no significant difference in LDL-C levels. At 90 days, all three biochemical parameters exhibited no significant statistical difference between the groups (Table II).

Additionally, all patients on the KD exhibited a moderate decrease in fasting glucose levels, although this was without statistical significance. At the end of the study, all patients on KD were within normoglycemic levels (Table III).

At the beginning of the study, the classification of nutritional status in the KD group according to body mass index (BMI) demonstrated that there was a predominance of overweight patients $(n=4 ; 44 \%)$, followed by patients in the normal range $(n=3 ; 33 \%)$ and patients with grade I obesity $(n=2 ; 22 \%)$, both at baseline and at the end of the study. One patient exhibited a worsening of nutritional status, evolving from overweight to obesity grade I following 90 days of KD, and one patient had an improvement in nutritional status, from grade I obesity to overweight (Table IV).

\section{Discussion}

The lack of effective treatments for patients with malignant glioma requires the design of novel therapeutic strategies that take advantage of common glioma phenotypes, including the altered metabolism of glioma cells (23). It has been postulated that a ketogenic diet, i.e., a diet consisting of high fat, low carbohydrates and adequate protein, may be useful in the treatment of brain tumors (25-31). The present study was conducted to assess the therapeutic efficacy and safety of a KD concomitant with intranasal perillyl alcohol as a novel therapeutic strategy for recurrent GBM. Following three months of adherence to the treatment, the following were observed: i) The PR rate was $77.8 \%$ (7/9 patients), including the reduction of the MRI tumor image and peritumoral edema; ii) reduced serum lipid levels; and v) decreased LDL-C levels.
Table I. General characteristics of KD and control groups.

\begin{tabular}{lcc}
\hline Characteristic & KD group & Control group \\
\hline Total, n & 9 & 8 \\
Age, years & 53 & 48 \\
Median & $31-61$ & $27-55$ \\
Range & & \\
Sex, n (\%) & $4(44)$ & $6(75)$ \\
Male & $5(56)$ & $2(25)$ \\
Female & & \\
Previous therapy, n & 9 & 8 \\
Radiotherapy & 9 & 8 \\
Chemotherapy & 9 & 8 \\
Anti-epileptic drug ${ }^{\mathrm{a}}$ & 9 & 8 \\
Corticosteroid & & \\
Karnofsky performance status & 0 & 1 \\
100 & 1 & 4 \\
90 & 6 & 3 \\
80 & 2 & \\
70 & &
\end{tabular}

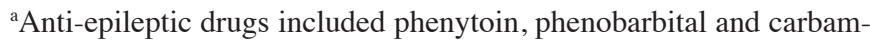
azepine. KD, ketogenic diet.

Kambach et al (40) demonstrated that glioma cells are sensitive to cholesterol synthesis inhibition downstream of the mevalonate pathway, suggesting that specifically targeting cholesterol synthesis could be an adjuvant treatment for GBM. Another study (41) proposed a potential molecular mechanism by which cholesterol reduction may be effective for preventing and treating the progression of malignant tumors; it suggested that upregulation of the cholesterol pathway may be associated with a poor prognosis in patients with GBM. Consistent with this model, the data of the present study indicated that there is a statistically significant difference in total cholesterol levels prior to subsequent to treatment with a KD with concomitant intranasal $\mathrm{POH}$, with lower values at the end of treatment (Fig. 3). However, no such difference was observed in the standard diet group. Furthermore, there was a statistically significant difference in LDL-C and triglyceride levels prior to subsequent to treatment in the KD group, with higher values at the onset (Table II).

In our previous study, it was demonstrated that although the consistent use of intranasal POH in recurrent GBM patients induced clinical responses, MRI data presented no evidence for the regression in the extent of peritumoral edema (42). In the present study, the extent of peritumoral edema was notably reduced, along with a decrease of the tumor area (Figs. 1 and 2). These results are consistent with another study (43) that investigated the effects of the KD on tumor growth and progression in animal models. The results revealed that peritumoral edema was significantly reduced in animals fed the KD, and protein analyses showed altered expression of zona occludens-1 and aquaporin-4. The authors concluded that the KD directly or indirectly altered the expression of several proteins involved in malignant 
Table II. Statistical comparison of biochemical parameters within and between the KD and control treatment groups.

\begin{tabular}{|c|c|c|c|c|c|c|c|}
\hline \multirow[b]{2}{*}{ Parameter } & \multicolumn{3}{|c|}{ KD group } & \multicolumn{3}{|c|}{ Control group } & \multirow{2}{*}{$\begin{array}{c}\text { P-value } \\
\text { between } \\
\text { KD and control }\end{array}$} \\
\hline & Mean \pm SD & Range & P-value & Mean \pm SD & Range & $\mathrm{P}$-value & \\
\hline \multicolumn{8}{|c|}{ Total cholesterol } \\
\hline Baseline & $260.9 \pm 51.5$ & $211-360$ & $0.003^{\mathrm{a}}$ & $195.6 \pm 42.7$ & $131-253$ & 0.663 & $0.013^{\mathrm{b}}$ \\
\hline After 90 days & $220.7 \pm 45.5$ & $170-320$ & & $192.6 \pm 48.0$ & $140-268$ & & 0.235 \\
\hline \multicolumn{8}{|c|}{ Low-density lipoprotein cholesterol } \\
\hline Baseline & $148.8 \pm 34.1$ & $103-200$ & $0.0002^{\mathrm{a}}$ & $118.6 \pm 36.8$ & $60-160$ & 0.642 & 0.100 \\
\hline After 90 days & $122.1 \pm 27.9$ & $89-172$ & & $116.5 \pm 36.0$ & $73-167$ & & 0.722 \\
\hline \multicolumn{8}{|l|}{ Triglycerides } \\
\hline Baseline & $180.4 \pm 100.9$ & $81-413$ & $0.004^{\mathrm{a}}$ & $100.4 \pm 64.8$ & $40-251$ & 1.000 & $0.036^{\mathrm{b}}$ \\
\hline After 90 days & $154.4 \pm 93.3$ & $71-373$ & & $99.1 \pm 42.0$ & $55-173$ & & 0.114 \\
\hline
\end{tabular}

${ }^{\mathrm{a}} \mathrm{P}<0.05,{ }^{\mathrm{b}} \mathrm{P}<0.01 . \mathrm{KD}$, ketogenic diet; $\mathrm{SD}$, standard deviation.

A

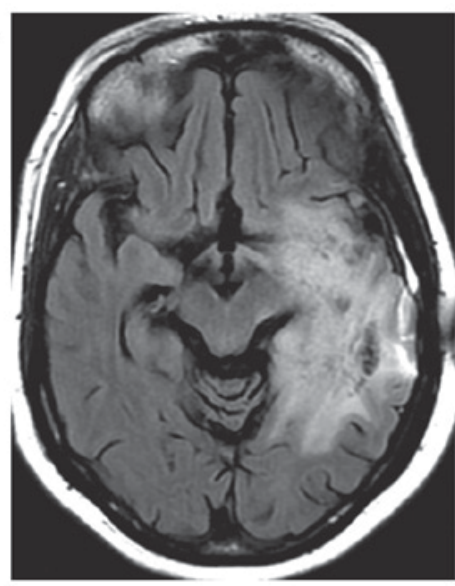

D

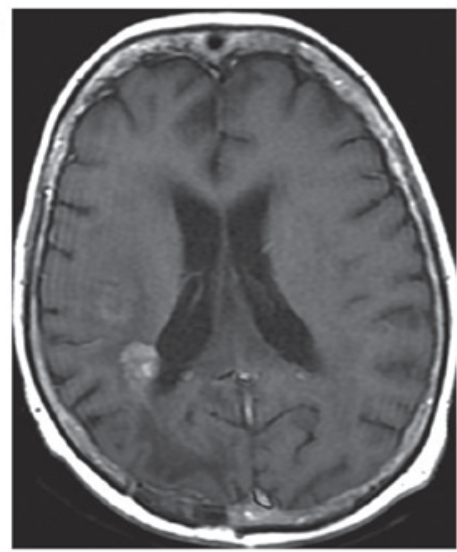

B

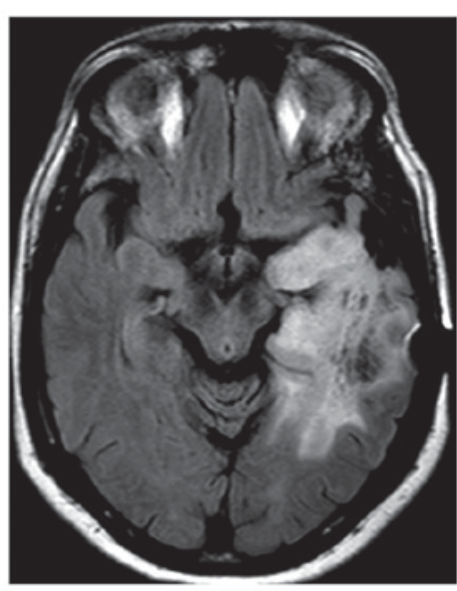

E

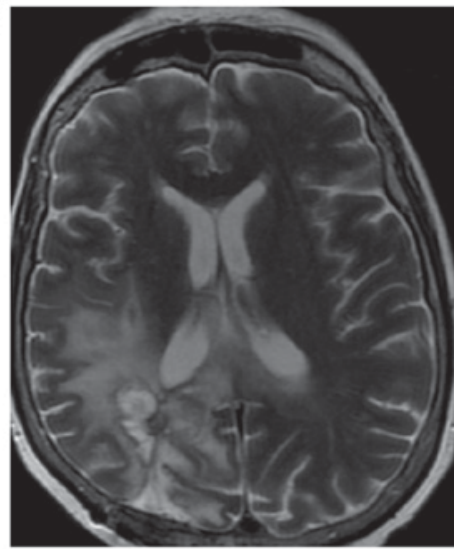

C

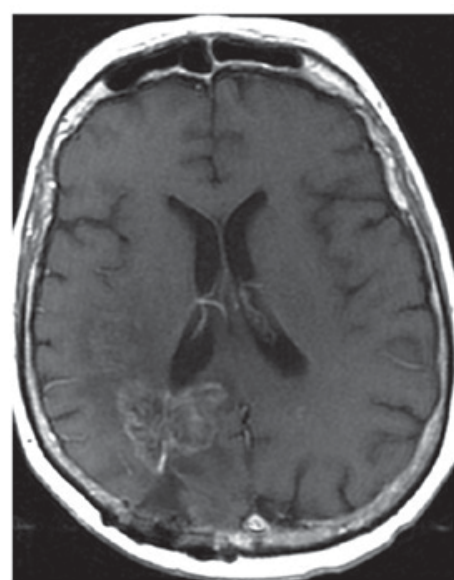

F

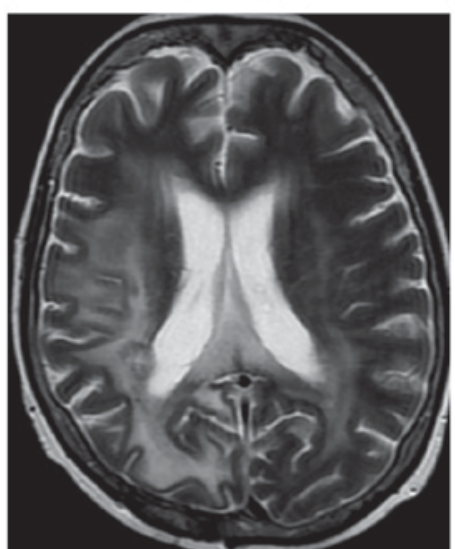

Figure 1. Brain magnetic resonance images of three patients, with images prior to (A,C,E) and following (B,D,F) the commencement of treatment. (A) Axial flair shows part of the expansive lesion and the extensive perilesional edema in the left temporoparietal region, which extends along the left cerebral peduncle. (B) Axial flair in the control shows reduction of lesion dimension. (C) Axial T1W contrast-enhanced image demonstrates lesion with peripheral and irregular enhancement and perilesional edema. (D) Axial T1W contrast-enhanced image of the control with reduction of tumor volume following therapy. Postoperative changes in correspondence. (E) Axial flair demonstrates a heterogeneous lesion with hyperintense area. (F) Axial flair of the control shows reduction in the dimensions of the heterogeneous lesion with extensive perilesional edema. Magnetic susceptibility artefact by previous biopsy.

progression and may be a useful tool for the treatment of gliomas. In addition to the MRI-based observation of decreased cerebral edema and tumor volume, a significant decrease in cholesterol and cholesterol esters in particles of LDL was identified in the present study. This observation is in line with an earlier study that demonstrated that proliferating tumor cells utilize serum-derived cholesterol esters, which are presumably carried by LDL particles (44). 
Table III. Biochemical parameters of the KD group prior and subsequent to 90 days of the KD.

A, Prior to the KD

\begin{tabular}{|c|c|c|c|c|c|}
\hline \multirow[b]{2}{*}{ PI } & \multicolumn{5}{|c|}{ Parameter, mg/dl } \\
\hline & $\mathrm{FG}$ & $\mathrm{TC}$ & LDL & HDL & $\mathrm{TG}$ \\
\hline 1 & 77 & 211 & 113 & 80 & 92 \\
\hline 2 & 71 & 295 & 189 & 79 & 136 \\
\hline 3 & 87 & 214 & 103 & 65 & 230 \\
\hline 4 & 96 & 306 & 147 & 145 & 81 \\
\hline 5 & 86 & 235 & 132 & 67 & 178 \\
\hline 6 & 80 & 360 & 200 & 69 & 413 \\
\hline 7 & 112 & 215 & 134 & 39 & 211 \\
\hline 8 & 90 & 275 & 182 & 70 & 116 \\
\hline 9 & 90 & 237 & 139 & 65 & 167 \\
\hline Mean \pm SD & $87.7 \pm 11.2$ & $260.9 \pm 49.0$ & $148.8 \pm 32.2$ & $75.4 \pm 27.0$ & $180 \pm 95.2$ \\
\hline
\end{tabular}

B, 3 months from the start of the KD

\begin{tabular}{|c|c|c|c|c|c|}
\hline \multirow[b]{2}{*}{ PI } & \multicolumn{5}{|c|}{ Parameter, mg/dl } \\
\hline & FG & $\mathrm{TC}$ & LDL & HDL & TG \\
\hline 1 & 74 & 170 & 93 & 61 & 81 \\
\hline 2 & 68 & 210 & 135 & 70 & 100 \\
\hline 3 & 82 & 211 & 89 & 70 & 200 \\
\hline 4 & 83 & 222 & 127 & 57 & 71 \\
\hline 5 & 81 & 200 & 120 & 73 & 134 \\
\hline 6 & 75 & 320 & 172 & 74 & 373 \\
\hline 7 & 95 & 188 & 100 & 50 & 190 \\
\hline 8 & 80 & 265 & 153 & 91 & 106 \\
\hline 9 & 85 & 200 & 110 & 70 & 135 \\
\hline Mean \pm SD & $83.4 \pm 13.03$ & $220.7 \pm 42.9$ & $122.1 \pm 26.3$ & $68.4 \pm 11.03$ & $154.4 \pm 87.9$ \\
\hline
\end{tabular}

$\mathrm{C}$, Normal range of parameters

\begin{tabular}{llllll}
\hline Parameter, mg/dl & FG & TC & LDL & HDL & TG \\
\hline Normal range & $<100$ & $<240$ & $<160$ & $>60$ & $<200$ \\
\hline
\end{tabular}

KD, ketogenic diet; PI, patient identifier; FG, fasting glucose; TC, LDL, low-density lipoprotein cholesterol; HDL, high-density lipoprotein cholesterol; TG, triglycerides; TC, total cholesterol.

The decrease of cholesterol in the present study can be explained as there was attention to the quality of the lipids offered (monounsaturated fatty acid $>$ polyunsaturated $>$ saturated fatty acid) and the amount of cholesterol was limited to $200 \mathrm{mg} /$ day. These results are not consistent with other studies (44-46), which concluded that KD increased the serum levels of total lipids and LDL-C. However, Sharma et al (47) did not observe significant alterations to the lipid profile of children with refractory epilepsy treated with KD, which remained within normal limits throughout the study.
In the present study, a moderate decrease in fasting glucose levels was documented, although without statistical significance (Table IV). The reason for this discrepancy is unclear and further investigation of this aspect will be required. It is important to note that the results of the present study were obtained with a 90-day period of dietary intervention. It is conceivable that a longer duration of the concomitant regimen may provide additional insight into the benefits of this regimen. However, shorter interventions may prove beneficial as well; for example, using a type 2 diabetic mouse model, Zhang et al (45) demonstrated that just 
Table IV. Anthropometric parameters and nutritional status of the KD treatment group.

\begin{tabular}{|c|c|c|c|c|c|c|c|c|}
\hline \multirow[b]{2}{*}{ PI } & \multirow[b]{2}{*}{ Height, $\mathrm{cm}$} & \multirow[b]{2}{*}{ Sex } & \multicolumn{3}{|c|}{ Before KD } & \multicolumn{3}{|c|}{ After KD } \\
\hline & & & $\mathrm{BM}, \mathrm{kg}$ & BMI, $\mathrm{kg} / \mathrm{m}^{2}$ & Rating & $\mathrm{BM}, \mathrm{kg}$ & BMI, $\mathrm{kg} / \mathrm{m}^{2}$ & Rating \\
\hline 1 & 1.68 & M & 79.8 & 28.29 & Overweight & 82.3 & 29.18 & Overweight \\
\hline 2 & 1.61 & $\mathrm{M}$ & 86.6 & 33.43 & Obese & 81.0 & 31.24 & Obese \\
\hline 3 & 1.66 & $\mathrm{M}$ & 81.8 & 29.74 & Overweight & 80.0 & 29.03 & Overweight \\
\hline 4 & 1.79 & M & 79.8 & 24.93 & Normal range & 76.4 & 23.87 & Normal range \\
\hline 5 & 1.68 & $\mathrm{M}$ & 79.8 & 28.29 & Overweight & 82.3 & 29.18 & Overweight \\
\hline 6 & 1.70 & F & 68.7 & 23.77 & Normal range & 65.7 & 22.73 & Normal range \\
\hline 7 & 1.79 & $\mathrm{~F}$ & 79.8 & 24.93 & Normal range & 76.4 & 23.87 & Normal range \\
\hline 8 & 1.87 & F & 100.9 & 28.91 & Overweight & 113.4 & 32.49 & Obese \\
\hline 9 & 1.78 & $\mathrm{~F}$ & 102.0 & 32.27 & Obese & 94.5 & 29.81 & Overweight \\
\hline Median & 1.70 & - & 81.8 & 29.69 & - & 81.0 & 29.16 & - \\
\hline
\end{tabular}

KD, ketogenic diet; PI, patient identifier; BM, body mass; BMI, body mass index.

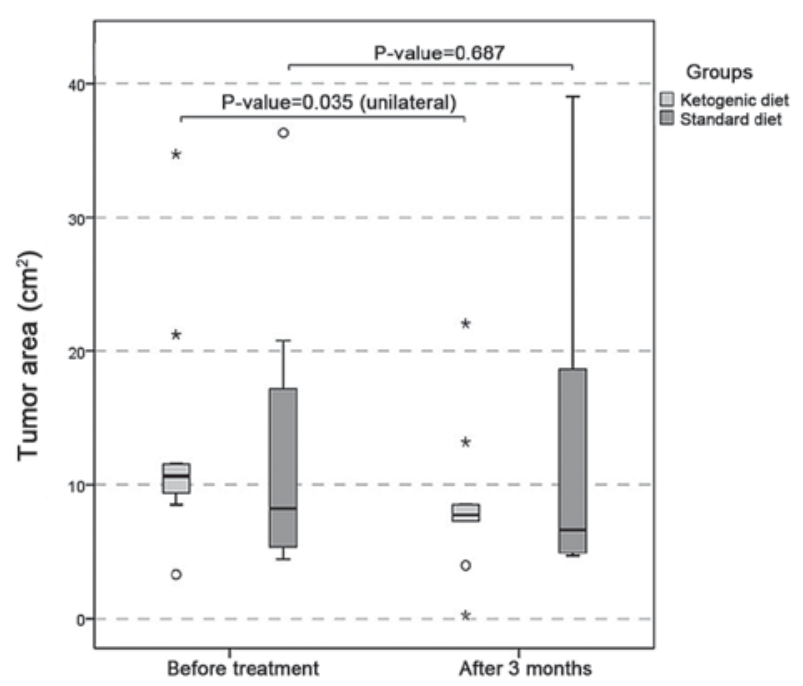

Figure 2. Tumor area $\left(\mathrm{cm}^{2}\right)$. Tumor area was significantly smaller in the ketogenic diet group at 90 days compared with the baseline $(\mathrm{P}=0.035)$, but not in the control group $(\mathrm{P}=0.687)$.

8 weeks of KD was sufficient to improve glucose and insulin tolerance, although hepatic lipid accumulation and hepatic steatosis were observed. Future studies investigating the KD as an adjuvant strategy to combat malignant gliomas should explore the optimal duration of this dietary intervention to support superior therapeutic outcomes.

In summary, the results of the present study are encouraging and suggest that KD associated with intranasal $\mathrm{POH}$ may represent a viable option as an adjunct therapy for recurrent GBM. Currently established therapeutic approaches for this patient cohort are lacking comprehensive efficacy, and the vast majority of patients succumb to the disease within a few years. Clearly, more effective treatments are urgently required. This small study will hopefully fuel further discussion and stimulate additional investigations to explore the potential benefit of adding a KD to existing regimens for patients with GBM.

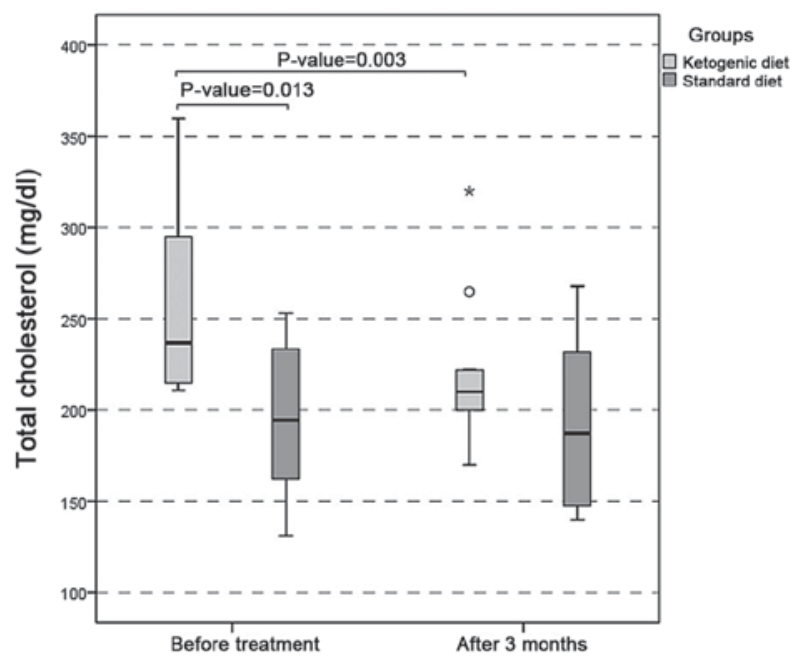

Figure 3. Total cholesterol (mg/dl). There was a statistically significant difference $(\mathrm{P}=0.003)$ between the total cholesterol level in the initial and final evaluations of the ketogenic diet group, with values significantly lower at 90 days.

\section{Acknowledgements}

The authors would like to thank Professor Licinio da Silva of the Mathematical Institute of the Fluminense Federal University for his technical assistance and statistical analysis.

\section{References}

1. Stupp R, Mason WP, van der Bent MJ, Weller M, Fisher B, Taphoorn MJ, Belanger $\mathrm{K}$, Brandes AA, Marosi C, Bogdahn U, et al: Radiotherapy plus concomitant and adjuvant temozolomide for glioblastoma. N Engl J Med 352: 987-996, 2005.

2. Hegi ME, Diserens AC, Gorlia T, Hamou MF, De Tribolet N, Weller M, Kros JM, Hainfellner JA, Mason W, Mariani L, et al: MGMT gene silencing and benefit from temozolomide in glioblastoma. N Engl J Med 352: 997-1003, 2005. 
3. Hegi ME, Liu L, Herman JG, Stupp R, Wick W, Weller M, Mehta MP and Gilbert MR: Correlation of O6-methylguanine methyltransferase (MGMT) promoter methylation with clinical outcomes in glioblastoma and clinical strategies to modulate MGMT activity. J Clin Oncol 26: 4189-4199, 2008.

4. Stupp R, Hegi ME, Mason WP, van den Bent MJ, Taphoorn MJ, Janzer RC, Ludwin SK, Allgeier A, Fisher B, Belanger K, et al: Effects of radiotherapy with concomitant and adjuvant temozolomide versus radiotherapy alone on survival in glioblastoma in a randomised phase III study: 5-year analysis of the EORTC-NCIC trial. Lancet Oncol 10: 459-466, 2009.

5. Schuster J, Lai RK, Recht LD, Reardon DA, Paleologos NA, Groves MD, Mrugala MM, Jensen R, Baehring JM, Sloan A, et al: A phase II, multicenter trial of rindopepimut (CDX-110) in newly diagnosed glioblastoma: The ACT III study. Neuro Oncol 17: 854-861, 2015

6. Narita Y: Bevacizumab for glioblastoma. Ther Clin Risk Manag 11: 1759-1765, 2015.

7. Reardon DA, Herndon JE II, Peters KB, Desjardins A, Coan A, Lou E, Sumrall AL, Turner S, Lipp ES, Sathornsumetee S, et al Bevacizumab continuation beyond initial bevacizumab progres sion among recurrent glioblastoma patients. Br J Cancer 107 $1481-1487,2012$

8. Abrams DA, Hanson JA, Brown JM, Hsu FP, Delashaw JB Jr and Bota DA: Timing of surgery and bevacizumab therapy in neurosurgical patients with recurrent high grade glioma. J Clin Neurosc 22: 35-39, 2015.

9. Swanson KD, Lok E and Wong ET: An overview of alternating electric fields therapy (NovoTTF Therapy) for the treatment of malignant Glioma. Curr Neurol Neurosci Rep 16: 8, 2016.

10. Stupp R, Taillibert S, Kanner AA, Kesari S, Steinberg DM, Toms SA, Taylor LP, Lieberman F, Silvani A, Fink KL, et al: Maintenance therapy with tumor-treating fields plus temozolomide vs temozolomide alone for glioblastoma: A randomized clinical trial. JAMA 314: 2535-2543, 2015

11. Chen TC, Da Fonseca CO and Schönthal AH: Preclinical development and clinical use of perillyl alcohol for chemoprevention and cancer therapy. Am J Cancer Res 5: 1580-1593, 2015.

12. da Fonseca CO, Schwartsmann G, Fischer J, Nagel J, Futuro D, Quirico-Santos T and Gattass CR: Preliminary results from a phase I/II study of perillyl alcohol intranasal administration in adults with recurrent malignant gliomas. Surg Neurol 70: 259-267, 2008.

13. da Fonseca CO, Simão M, Lins IR, Caetano RO, Futuro D and Quirico-Santos T. Efficacy of monoterpene perillyl alcohol upon survival rate of patients with recurrent glioblastoma. J Cancer Res Clin Oncol 137: 287-293, 2011.

14. Da Fonseca CO, Teixeira RM, Silva JC, DE Saldanha DA Gama Fischer J, Meirelles OC, Landeiro JA and Quirico-Santos T: Long-term outcome in patients with recurrent malignant glioma treated with Perillyl alcohol inhalation. Anticancer Res 33 5625-5631, 2013

15. Cross H: Epilepsy: Behavioural, psychological, and ketogenic diet treatments. BMJ Clin Evid 2015: pii: 1214, 2015.

16. Rho JM: How does the ketogenic diet induce anti-seizure effects? Neurosci Lett 637: 4-10, 2017.

17. Bruna J, Miró J and Velasco R: Epilepsy in glioblastoma patients: Basic mechanisms and current problems in treatment. Expert Rev Clin Pharmacol 6: 333-344, 2013.

18. Kargiotis O, Markoula S and Kyritsis AP: Epilepsy in the cancer patient. Cancer Chemother Pharmacol 67: 489-501, 2011.

19. Armstrong TS, Grant R, Gilbert MR, Lee JW and Norden AD: Epilepsy in glioma patients: Mechanisms, management, and impact of anticonvulsant therapy. Neuro Oncol 18: 779-789, 2016

20. Liberti MV and Locasale JW: The warburg effect: How does it benefit cancer cells? Trends Biochem Sci 41: 211-218, 2016.

21. Kroemer G and Pouyssegur J: Tumor cell metabolism: Cancer's Achilles' heel. Cancer Cell 13: 472-482, 2008.

22. Lu J, Tan M and Cai Q: The Warbu'rg effect in tumor progression: Mitochondrial oxidative metabolism as an anti-metastasis mechanism. Cancer Lett 356: 156-164, 2015.

23. Seyfried TN, Flores R, Poff AM, D'Agostino DP and Mukherjee P: Metabolic therapy: A new paradigm for managing malignant brain cancer. Cancer Lett 356: 289-300, 2015.

24. Bhattacharya B, Mohd Omar MF and Soong R: The Warburg effect and drug resistance. Br J Pharmacol 173: 970-979, 2016.

25. Woolf EC, Syed N and Scheck AC: Tumor metabolism, the ketogenic diet and -hydroxybutyrate: Novel approaches to adjuvant brain tumor therapy. Front Mol Neurosci 9: 122, 2016.

26. Klement RJ, Champ CE, Otto C and Kämmerer U: Anti-tumor effects of ketogenic diets in mice: A meta-analyais. PLoS One 11: e0155050, 2016.
27. Abdelwahab MG, Fenton KE, Preul MC, Rho JM, Lynch A, Stafford P and Scheck AC: The ketogenic diet is an effective adjuvant to radiation therapy for the treatment of malignant glioma. PLoS One 7: e36197, 2012.

28. Maroon JC, Seyfried TN, Donohue JP and Bost J: The role of metabolic therapy in treating glioblastoma multiforme. Surg Neurol Int 6: 61, 2015.

29. Zuccoli G, Marcello N, Pisanello A, Servadei F, Vaccaro S, Mukherjee P and Seyfried TN: Metabolic management of glioblastoma multiforme using standard therapy together with a restricted ketogenic diet: Case report. Nutr Metab (Lond) 7: 33 , 2010.

30. Champ CE, Palmer JD, Volek JS, Werner-Wasik M, Andrews DW, Evans JJ, Glass J, Kim L and Shi W: Targeting metabolism with a ketogenic diet during the treatment of glioblastoma multiforme. J Neurooncol 117: 125-131, 2014.

31. Schwartz K, Chang HT, Nikolai M, Pernicone J, Rhee S, Olson K, Kurniali PC, Hord NG and Noel M: Treatment of glioma patients with ketogenic diets: Report of two cases treated with an IRB-approved energy-restricted ketogenic diet protocol and review of the literature. Cancer Metab 3: 3, 2015.

32. Wagner M and Moore DD: Endoplasmic reticulum stress and glucose metabolism. Curr Opin Clin Nutr Metab Care 14: 367-373, 2011.

33. Schönthal AH, Chen TC, Hofman FM, Louie SG and Petasis NA Preclinical development of novel anti-glioma drugs targeting the endoplasmatic reticulum stress response. Curr Pharm Des 17: 2428-2438, 2011.

34. Kato $\mathrm{H}$ and Nishitoh H: Stress responses from the endoplasmic reticulum in cancer. Front Oncol 5: 93, 2015.

35. Peñaranda Fajardo NM, Meijer C and Kruyt FA: The endoplasmatic reticulum stress/unfolded protein responde in gliomagenesis, tumor progression and as a therapeutic target in glioblastoma. Biochem Pharmacol 118: 1-8, 2016.

36. Johnson GG, White MC and Grimaldi M: Stressed to death: Targeting endoplasmic reticulum stress response induced apoptosis in gliomas. Curr Pharm Des 17: 284-292, 2011.

37. Cho HY, Wang W, Jhaveri N, Torres S, Tseng J, Leong MN, Lee DJ, Goldkorn A, Xu T, Petasis NA, et al: Perillyl alcohol for the treatment of temozolomide-resistant gliomas. Mol Cancer Ther 11: 2462-2472, 2012.

38. Schonthal AH: Targeting endoplasmic reticulum stress for cancer therapy. Front Biosci (Schol Ed) 4: 412-431, 2012.

39. Strowd RE III and Grossman SA: The role of glucose modulation and dietary supplementation in patients with central nervous system tumors. Curr Treat Options Oncol 16: 36, 2015.

40. Kambach DM, Halim AS, Cauer AG, Sun Q, Tristan CA, Celiku O, Kesarwala AH, Shankavaram U, Batchelor E and Stommel JM: Disabled cell density sensing leads to dysregulated cholesterol synthesis in glioblastoma. Oncotarget 8: 14860-14875, 2017.

41. Murai T, Maruyama Y, Mio K, Nishiyama H, Suga M and Sato C: Low cholesterol triggers membrane microdomain-dependent CD44 shedding and suppresses tumor cell migration. J Biol Chem 286: 1999-2007, 2011.

42. Da Fonseca CO, Silva JT, Lins IR, Simão M, Arnobio A, Futuro D and Quirico-Santos T: Correlation of tumor topography and peritumoral edema of recurrent malignant gliomas with therapeutic response to intranasal administration of perillyl alcohol. Invest New Drugs 27: 557-564, 2009.

43. Woolf EC, Curley KL, Liu Q, Turner GH, Charlton JA, Preul MC and Scheck AC: The ketogenic diet alters the hypoxic response and affects expression of proteins associated with angiogenesis, invasive potential and vascular permeability in a mouse glioma model. PLoS One 10: e0130357,2015.

44. Nygren C, von Holst H, Månsson JE and Fredman P: Increased levels of cholesterol esters in glioma tissue and surrounding areas of human brain. Br J Neurosurg 11: 216-220, 1997.

45. Zhang X, Qin J, Zhao Y, Shi J, Lan R, Gan Y, Ren H, Zhu B, Qian M and Du B: Long-term ketogenic diet contributes to glycemic control but promotes lipid accumulation and hepatic steatosis in type 2 diabetic mice. Nutr Res 36: 349-358, 2016.

46. Zamani GR, Mohammadi M, Ashrafi MR, Karimi P, Mahmoudi M, Badv RS, Tavassoli AR and Azizi Malamiri R: The effects of classic ketogenic diet on serum lipid profile in children with refractory seizures. Acta Neurol Belg 116: 529-534, 2016.

47. Sharma S, Gulati S, Kalra V, Agarwala A and Kabra M: Seizure control and biochemical profile on the ketogenic diet in young children with refractory epilepsy-Indian experience. Seizure 18: 446-449, 2009. 